\title{
21st Century Approach to Splenic Injury
}

\section{Charles E Lucas}

\section{ABSTRACT}

Recent trends of splenic injury management in the 21st century emphasizes nonoperative treatment in children and adults. The clinician must base his treatment on clinical criteria on hemodynamic stability and associated injuries. The risk of overwhelming post-splenectomy sepsis is very low and should not influence the decision to operate, if the clinical condition demands it.

\section{Keywords: Splenic injury, OPSI, Trauma to spleen.}

How to cite this article: Lucas CE. 21st Century Approach to Splenic Injury. J Trauma Critical Care Emerg Surg 2013;2(3): 116-125.

\section{Source of support: Nil}

\section{Conflict of interest: None}

\section{RESUMEN}

Las últimas tendencias de la gestión de la lesión esplénica en el siglo 21 hace hincapié en el tratamiento no quirúrgico en niños y adultos. El médico debe basar su tratamiento en los criterios clínicos desde la estabilidad hemodinámica y lesiones asociadas. El riesgo de sepsis abrumadora post-esplenectomía es muy bajo y no debe influir en la decisión de operar, si la situación clínica lo exige.

Palabras claves: Lesión esplénica, OPSI, Traumatismo en el bazo.

\section{INTRODUCTION}

Splenic function has been an enigma for over two millennia. ${ }^{1,2}$ Aristotle proclaimed that the spleen has no purpose. The Babylonian Talmud reports that the spleen promotes laughter; Pliny, in $87 \mathrm{AD}$, ascribed humor to the spleen but warned that it slows runners. The 16th century scholars considered the spleen essential for life, whereas the great Malpighi, in the 17 th century, taught that the spleen was not needed. The 18th century consensus was that the spleen was a redundant organ. These philosophical proclamations had no clinical significance since splenic surgery was unknown. These teachings transcended the theoretical arena to practical application in 1897 when Riegner performed what many believe is the first splenectomy for trauma. ${ }^{2}$ The patient had a major injury, had suffered significant blood

\begin{tabular}{|l|l|}
\multicolumn{1}{|c|}{ Table 1: Splenic maturation } \\
\hline Birth & Immature function and histology \\
\hline Neonate & Lymph cells: no germ centers \\
\hline Puberty & Maximal weight $25 \%$ total lymphoid mass \\
\hline Maturity & $\begin{array}{l}\text { Leukocyte sequestration Ig and antibody } \\
\text { production para-follicular cells }\end{array}$ \\
\hline
\end{tabular}

loss, and recovered after splenectomy, thereby establishing the principle that splenectomy for injury saves lives.

\section{SPLENIC MATURATION}

The spleen, at birth, is functionally and histologically immature. ${ }^{1,2}$ During the neonatal years, lymph follicles form, but there are no germ centers. The spleen reaches its maximal weight during puberty when it represents $25 \%$ of total lymphoid mass. At maturity there is leukocyte sequestration, which is associated with immunoglobulin and antibiotic production (Table 1). The antibiotic response to foreign antigens comes from the parafollicular cells. These cells do not survive after splenic replantation. ${ }^{3}$

\section{FIRST HALF 20TH CENTURY}

Reigner's proclamation that splenectomy for trauma saves lives and causes no ill effects extended throughout the next 50 years. Pierce, in 1918, noted that the postsplenectomy state was associated with anemia, eosinophelia, and leukocytosis; $25 \%$ of his asplenic dogs died from pneumonia but, for reasons which are still unclear, he concluded that splenectomy is tolerated with 'relative impunity'. ${ }^{2}$ The following year, Morris and Bullock presented excellent scientific evidence that the spleen is important in combating infection. ${ }^{4}$ They noted that asplenic dogs subjected to a pasteurella challenge had a much greater susceptibility to infection compared to splenic controls. They concluded that the spleen enhances the immune response. Their conclusions

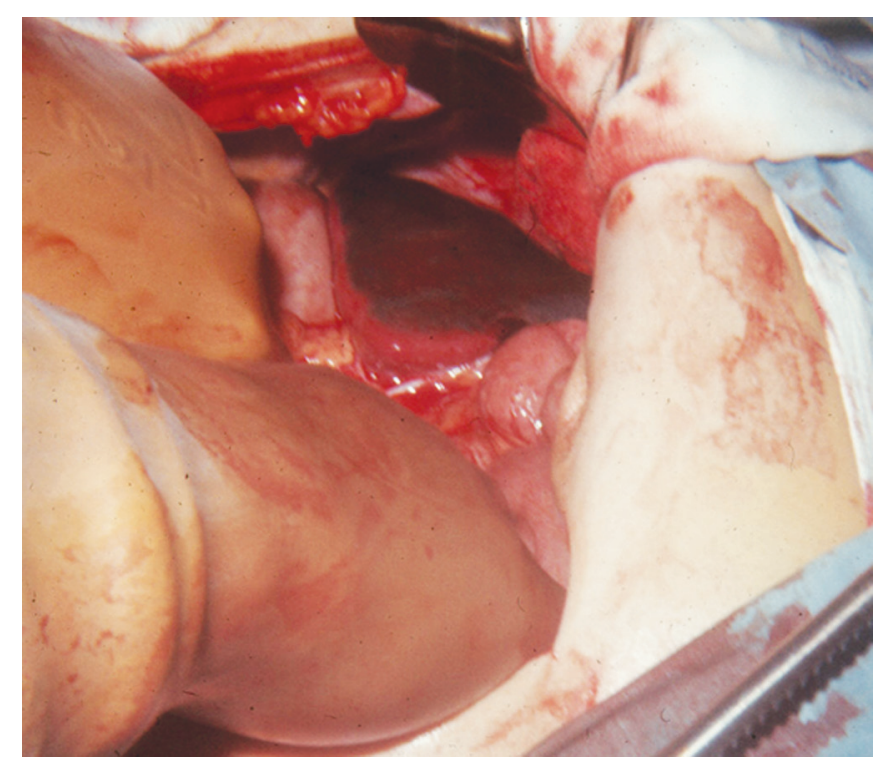

Fig. 1: An intrasplenic hematoma involving $30 \%$ of the spleen had to be removed in the 1970 s for fear of delayed rupture 
were ignored for the next generation. Famous surgeons suggested that fear of splenectomy was 'poppycock' while Pfieiffer, in 1924, was humiliated for suggesting that longterm data after splenectomy are needed. ${ }^{5,6}$

\section{DELAYED SPLENIC BLEEDING}

Based upon his experience with a single patient, MacIndoe, in 1932, proclaimed a 10 to $15 \%$ incidence of delayed splenic bleeding in patients in whom an early diagnosis of splenic injury was not made. ${ }^{7}$ He described how a hematoma within the substance of the spleen would expand from osmotically driven forces, so that the rising pressure within the hematoma would burst the splenic capsule leading to hemoperitoneum. All injured spleens, therefore, had to be removed (Fig. 1). This observation, of course, was made before the widespread use of diagnostic peritoneal lavage (DPL) which became popular 30 years later. The routine use of DPL showed that patients with a negative DPL almost never had 'delayed rupture of the spleen' during a period of nonoperative therapy. The widespread use of DPL identified that the socalled 'delayed splenic rupture' was really delayed diagnosis of an early splenic rupture. ${ }^{2}$ The author has seen only one patient who developed delayed hemoperitoneum of splenic origin following a negative DPL (Fig. 2).

\begin{tabular}{|c|c|}
\hline Grade & Injury description \\
\hline I & $\begin{array}{l}\text { Hematoma }<10 \% \text { surface area } \\
\text { Laceration }<1 \mathrm{~cm} \text { deep }\end{array}$ \\
\hline II & $\begin{array}{l}\text { Hematoma } 10-20 \% \text { surface area } \\
\text { Laceration } 1-3 \mathrm{~cm} \text { deep }\end{array}$ \\
\hline III & $\begin{array}{l}\text { Hematoma }>50 \% \text {, expanding hematoma } \\
\text { Active bleeding } \\
\text { Laceration }>3 \mathrm{~cm} \text { deep or involves trabecular vessels }\end{array}$ \\
\hline IV & $\begin{array}{l}\text { Ruptured or actively bleeding hematoma } \\
\text { Laceration to hilum; } 25 \% \text { ischemia }\end{array}$ \\
\hline V & Shattered or totally devascularized \\
\hline
\end{tabular}

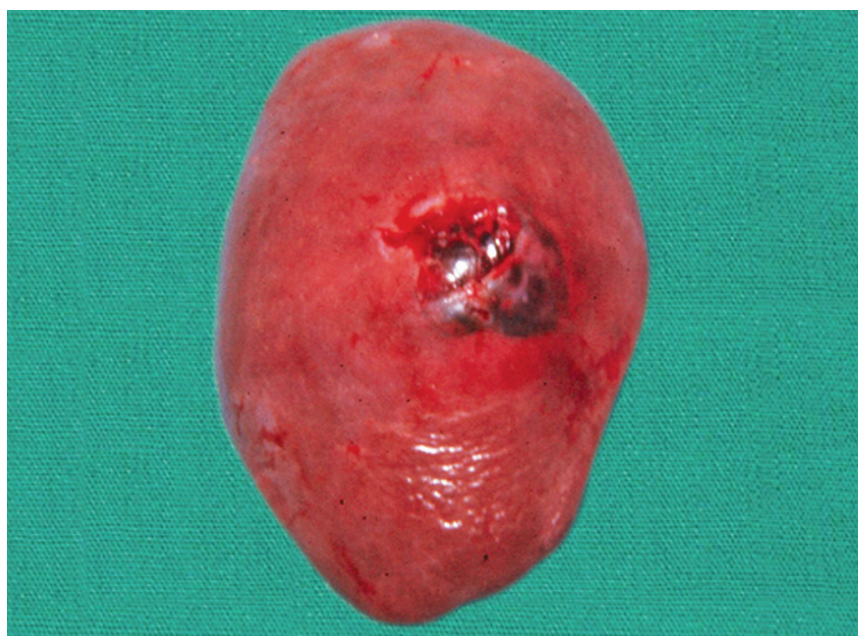

Fig. 2: This is the only splenic rupture diagnosed late after an early negative DPL. Presumably, this patient had a delayed rupture of the spleen which is very rare

\section{THE SPLEEN AND INFECTION}

The next serious challenge to the concept that the spleen performed no useful function came from King and Shumacker in 1952 when they reported a syndrome of overwhelming postsplenectomy infection (OPSI) in two of five children undergoing splenectomy for congenital spherocytosis. ${ }^{8}$ Shortly thereafter, other authors reported OPSI in patients with hemolytic diseases. ${ }^{9}$ Smith, in 1957 , reported the first case of OPSI following splenectomy in an adult with no pre-existent comorbidities. ${ }^{10}$ Despite this prescient report, the surgical community continued to do routine splenectomy for even the most minor splenic injuries.

The author was taught in the 1960s, and later taught his residents in the $1970 \mathrm{~s}$, to perform splenectomies for intrasplenic hematomas and minor stab wounds. ${ }^{2}$ Lower pole vessels which were avulsed during gastric mobilization, while doing truncal vagotomy led to splenectomy.

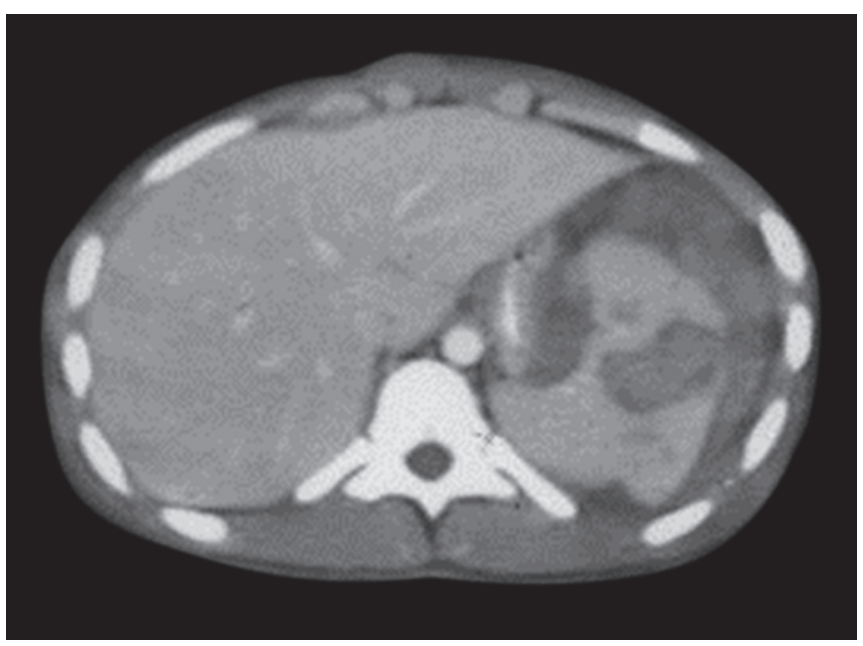

Fig. 3: An intrasplenic hematoma involving $20 \%$ of the surface area is a grade II injury. This patient was observed nonoperatively and discharged home on day 3

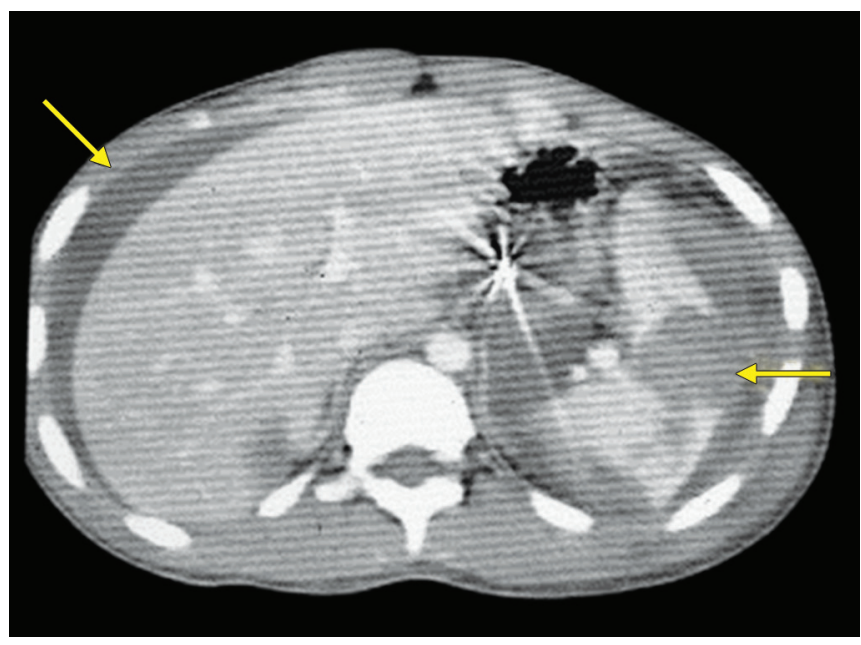

Fig. 4: A splenic rupture extending to the trabecular vessels is a grade III injury. This patient, with parahepatic and parasplenic blood, was treated nonoperatively and went home on day 4 
MacIndoe's concept of delayed splenic hemorrhage survived half a century! Like many others in the surgical community, the author ignored the prescient observation by Smith about OPSI following splenectomy for trauma in previously healthy adults. This confirms the old axiom: he who ignores history is doomed to repeat its errors.

\section{SPLENIC INJURY SEVERITY}

The classic categorization of the severity of splenic injury was developed in the 1980s and improved in the 1990s (Table 2). ${ }^{11}$ Grade I splenic injury is defined as an intrasplenic hematoma less than $10 \%$ of surface area or a splenic laceration less than $1 \mathrm{~cm}$ in depth. Grade II injury includes a hematoma involving 10 to $20 \%$ of surface area or a laceration 1 to $3 \mathrm{~cm}$ in depth (Fig. 3). Grade III injury includes a hematoma involving more than half of the spleen, or expanding with active bleeding, or a laceration which is more than $3 \mathrm{~cm}$ deep or involves the trabecular vessels (Fig. 4).

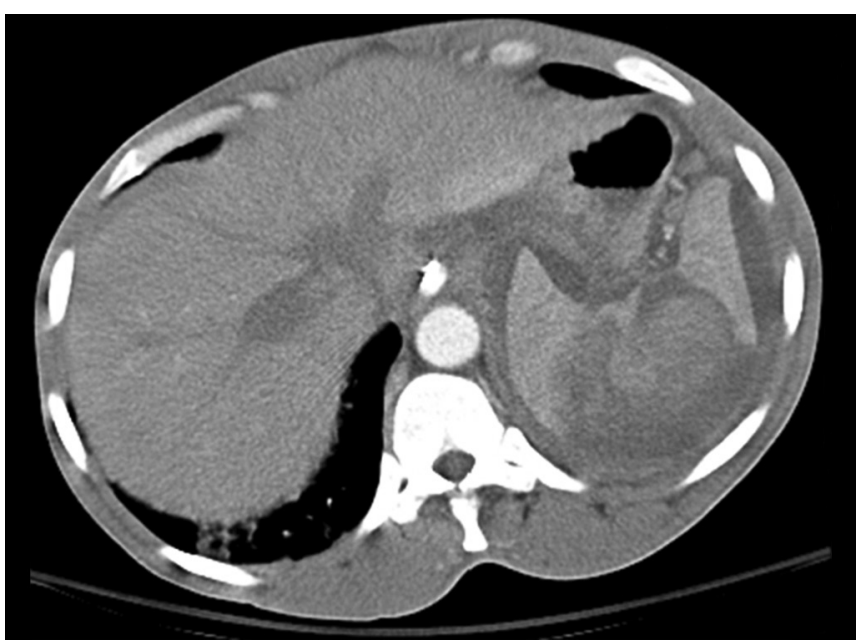

Fig. 5: This patient, with an injury extending through to the hilum, had a grade IV injury. Thirty-six hours after initiating nonoperative therapy, he developed left upper quadrant pain and underwent splenectomy

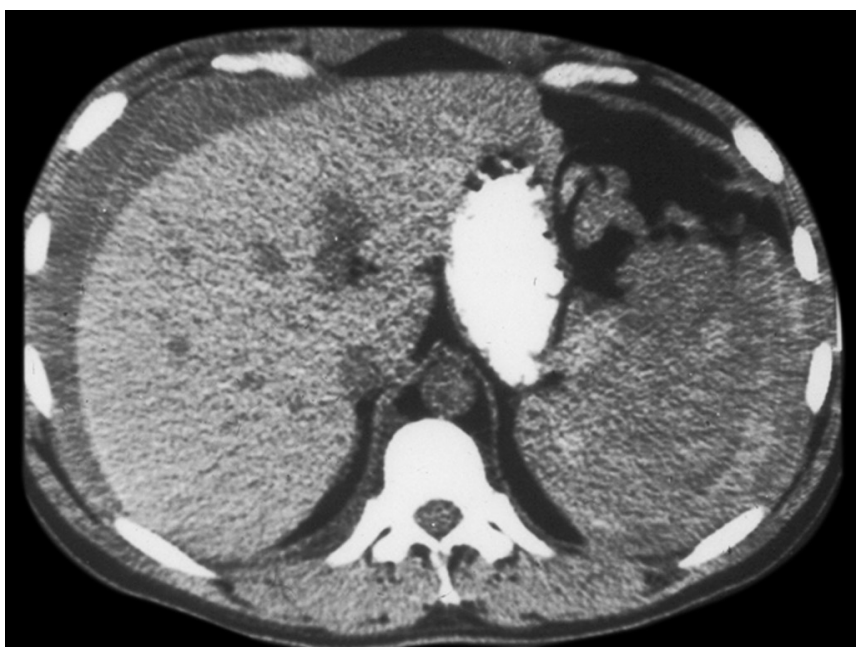

Fig. 6: This patient, with a fragmented grade $V$ splenic injury, underwent early splenectomy plus hepatorrhaphy for associated liver injury
Grade IV injury consists of a ruptured, actively bleeding splenic hematoma, a parenchymal laceration which extends to the hilum, or ischemia involving $25 \%$ of the spleen (Fig. 5). The grade $\mathrm{V}$ injury is a shattered or devascularized spleen (Fig. 6). Prior to the abbreviated injury score (AIS), the surgical community would talk about minor and major injuries which reflected the later formal description of these injuries.

\section{OPERATIVE SPLENIC SALVAGE: SPLENORRHAPHY}

The gradual acceptance that splenectomy was detrimental to immune function led to a number of operative techniques designed to preserve all or part of the spleen. Interestingly, Leo Dretzka, the first chief of surgery at the Detroit Receiving Hospital, published the first report on splenorrhaphy in a child in 1930; his contributions were ignored. ${ }^{2}$ The classic descriptions of splenorrhaphy in adults came from

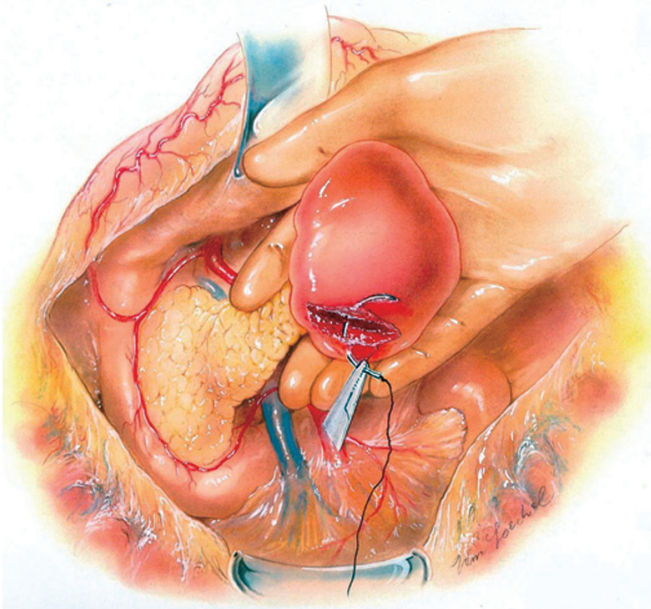

Fig. 7: Following wide exposure of the spleen and distal pancreas, the spleen is gently lifted with the pancreas into the wound in order to facilitate gentle splenorrhaphy

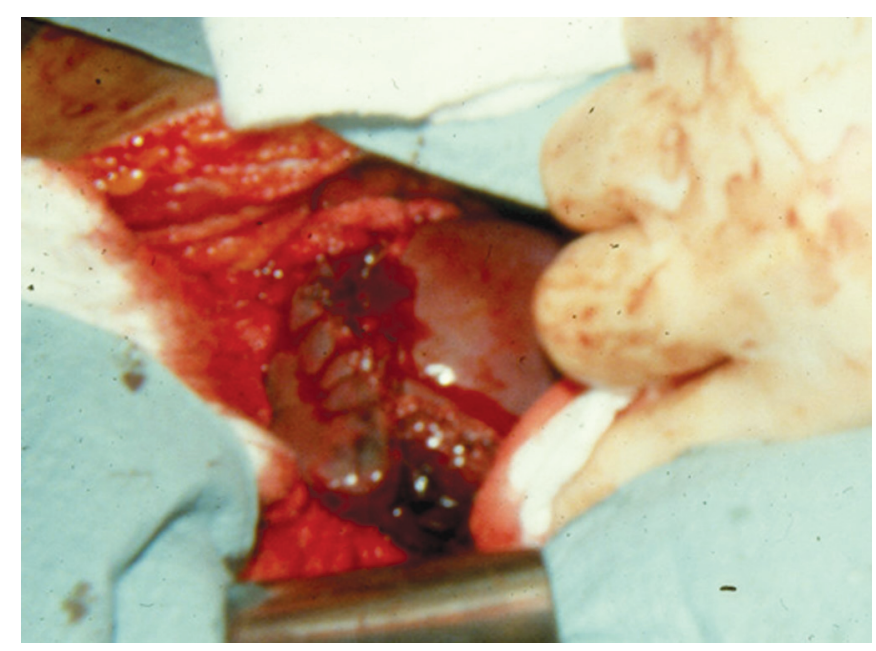

Fig. 8: During hemisplenectomy, the splenic capsule is preserved in order to cover the exposed parenchyma. The capsular wound healing is superior to parenchymal wound healing 
Morgenstern et al in 1979; they emphasized the importance of a generous incision, division of the greater omentum, careful retraction of the stomach, and gentle mobilization of the spleen with pancreas into the wound to facilitate direct placement of sutures (Fig. 7). This article emphasized a preference for using absorbable sutures for parenchyma hemostasis, permanent sutures for exposed bleeding vessels, resection of pulverized or ischemic segments, electrocautery for 'capillary' oozing, and preservation of splenic capsule for reattachment over a resected segment of spleen (Fig. 8). ${ }^{12,13}$ Numerous animal studies showed that splenic function was preserved in direct proportion to the splenic volume. ${ }^{14}$ A $75 \%$ splenectomy was better than total splenectomy but well below the asplenic state. Thus, partial splenectomy of ischemic or shattered fragments became popular in order to preserve partial immune function. ${ }^{15}$ During the late 1970s, the author performed successful splenorrhaphy or partial splenectomy in 12 of 13 consecutive patients; the failure was in a patient with portal hypertension which, we now know, is best treated with total splenectomy. Success with operative splenorrhaphy correlates closely with the AIS. Operative splenic salvage is successful in the vast majority of patients with minor (AIS I-III) injury and is less successful in patients actively bleeding from major (AIS IV-V) injury. ${ }^{16}$ In retrospect, the high success rate of obtaining operative hemostasis in patients with minor (AIS I-III) injuries probably reflects the fact that these injuries would probably have stopped bleeding and could have been treated ${ }^{1,2}$ nonoperatively.

\section{OPERATIVE SPLENIC SALVAGE: SPLENIC REPLANTATION}

The observation that patients with prior splenic rupture may later develop a syndrome of splenosis with multiple

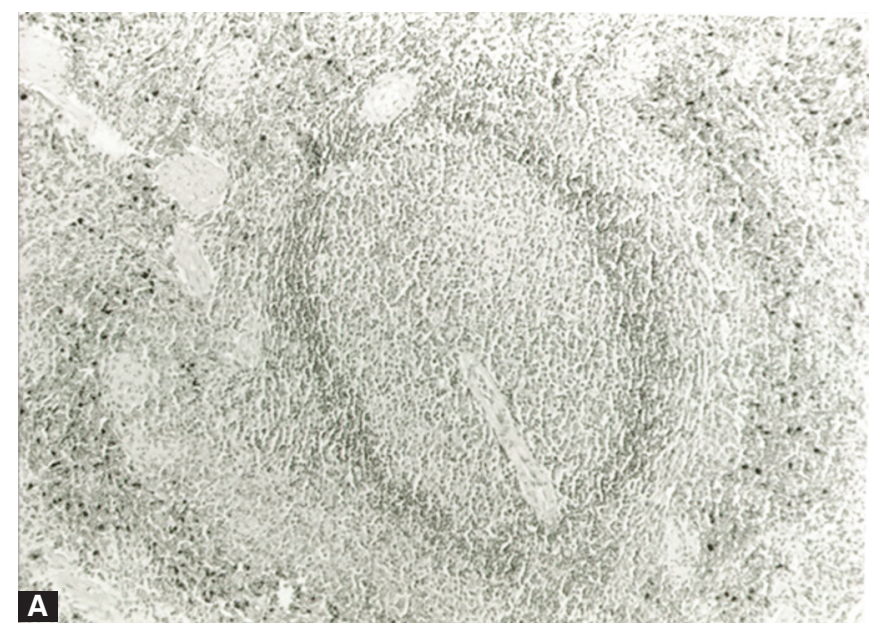

splenic fragments surviving in the peritoneal cavity raised a question as to whether a contained collection of splenic fragments might be an alternative method for splenic salvage when total splenectomy is needed. This led to the practice of dicing up the resected spleen to pieces less than $1 \mathrm{~cm}$ and placing them within the omentum, which is folded onto itself, thus resembling a 'splenic omelet'. ${ }^{17}$ This technique for replantation became popular and was widely recommended. Animal studies demonstrated that the replanted spleen would undergo cellular death but that new cells would form on the residual splenic matrix with splenic tissue being identifiable within six months. ${ }^{18,19}$ Survival of splenic tissue after replantation was also seen in man. The presumption was made that these regenerated splenic cells would exert their normal immune function. The author questioned the wisdom of replantation after replanting the resected spleen in a middle-aged woman who sustained an isolated type 4 blunt rupture. The replantation was done as described above. By postoperative day 4 , when most such patients should have been discharged home, this patient developed high leukocytosis and fever necessitating re-exploration. The splenic bed was normal, and the omental omelet with the replanted spleen was removed. One day later, she was afebrile, feeling well and discharged home two days later.

This complication was clearly different from prior splenectomies for single organ injury and led to an assessment of the replantation technique in an animal model. Dogs that had undergone splenectomy alone, or splenectomy with replantation, were compared to eusplenic dogs. ${ }^{3}$ These animals were followed for 18 months. The splenectomy dogs and the replanted dogs showed no uptake of technician labeled red cells at 10 months and no response to type III pneumococcus challenges at 9 months in contrast to eusplenic dogs Autopsy examinations at 18 months demonstrated that, indeed, the replanted

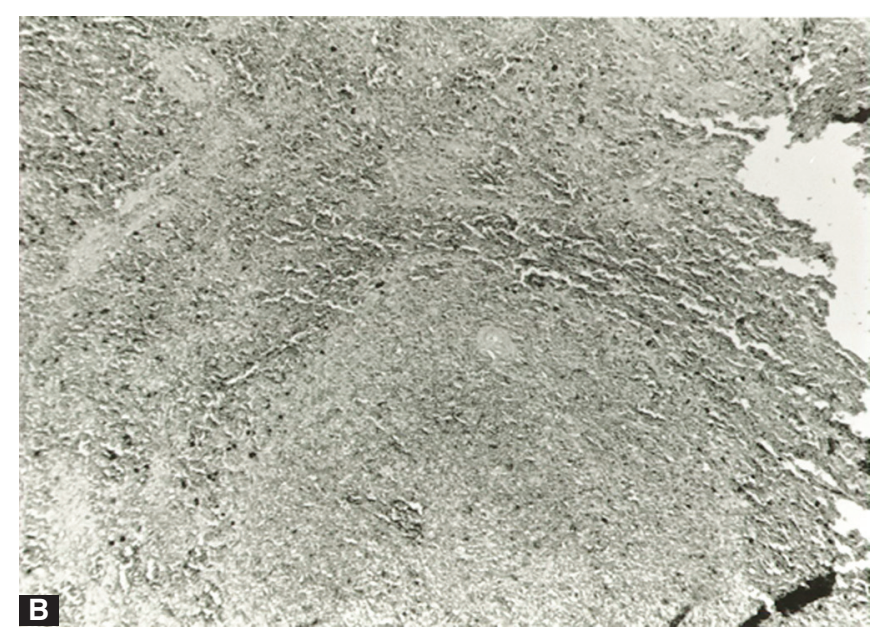

Figs 9A and B: Eighteen months following laparotomy without splenectomy, the normal spleens show lymphoid tissue and parafollicular cells around the germ centers $(A)$ whereas, the regenerated spleen within the 'splenic omelet' has extensive fibrosis without parafollicular cells surrounding the germ centers (B). The parafollicular cells are responsible for immunoglobulin and antibiotic generation 
animals had viable splenic tissues. The histologic studies, however, demonstrated that these transplanted spleens had no parafollicular lymphoid cells. The parafollicular pulp was fibrotic (Figs 9A and B). As indicated previously, these cells are responsible for the splenic immune response. None of the clinical publications on replanted spleens have shown parafollicular lymphoid cells. This negates the scientific basis upon which is based the concept of doing splenic replantation and explains the lack of benefit clinically. ${ }^{20}$ The author no longer recommends splenic replantation, which has fallen out of favor in the surgical community.

\section{SPLENIC WOUND HEALING AFTER SPLENORRHAPHY}

The widespread acceptance of splenorrhaphy raised the question as to whether the repaired spleen was prone to delayed rupture. Should the splenorrhaphy patient be kept at rest or be restricted from normal activities for a specific period of time? Splenic wound healing after splenorrhaphy and nonsuture pressure control was assessed in porcine and canine models. ${ }^{21}$ Injured actively bleeding spleens were made hemostatic by splenorrhaphy or pressure alone, the latter mimicking the patient treated nonoperatively. Both the sutured spleens and the compressed spleens had excellent breaking strength at three and 6 weeks; this strength exceeded that of midline fascial wound strength after laparotomy. Clearly, any decisions regarding restriction of going back to work should be related more to the fascial closure than the splenorrhaphy.

\section{SPLENIC SALVAGE: NONOPERATIVE OBSERVATION}

Billroth, in the 1870s, suggested that not all injured spleens must be removed; this observation was made at autopsy

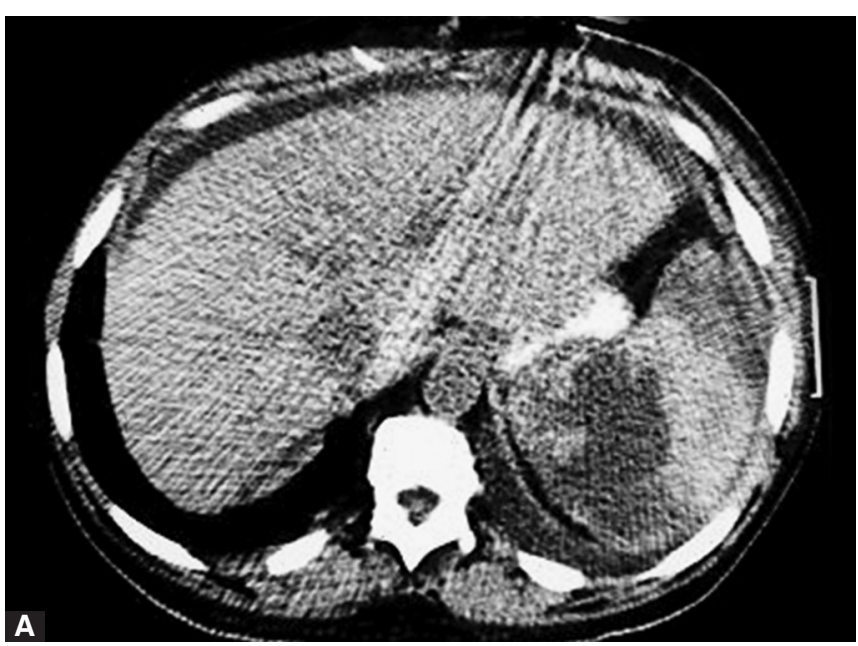

examination on a patient who died five days after head injury. ${ }^{1,2}$ An incidental ruptured spleen was surrounded by a coagulopathy with no recent bleeding. The general acceptance of operative splenic salvage led to the recognition by several surgeons that the bleeding had already stopped by the time of laparotomy; the addition of splenorrhaphy sometimes became meddlesome. Despite these preliminary observations, it was not until 1978 that Dr Tom et al at the William Beaumont Hospital (WBH) in Michigan instituted the first prospective protocol of planned nonoperative therapy (NOT). ${ }^{22}$ Surgeons agreeable to the protocol would provide NOT for all stable patients with blunt splenic rupture and no other reason for laparotomy. All patients so treated in their first 5 years did well, after which all of the surgeons bought into the NOT protocol. Their second 5-year report had only one patient selected for NOT who underwent exploration; this single patient probably did not need exploration but was the victim of a nervous attending surgeon who was not completely comfortable with the NOT protocol of splenic rupture; the patient never received a blood transfusion nor was hypotensive. ${ }^{23}$

These authors have continued to report their prospective findings in successive 5 -year periods; in the 21 st century, their success of NOT has risen to $80 \%$ of all patients admitted with blunt splenic rupture. ${ }^{24}$ A number of subsequent papers by many authors have confirmed these successful findings, so that NOT is the accepted therapy for stable patients with blunt splenic injury. For reasons unclear to the author, these subsequent papers somehow fail to cite the world's first series of NOT for blunt splenic injury. ${ }^{25,26}$ Later publications suggested NOT should be denied to patients over the age of 65 . The original studies from the WBH showed no age discrimination. The 21 st century recommendations indicate that patients of all ages who are stable and do not require blood transfusions are candidates for NOT. ${ }^{24}$

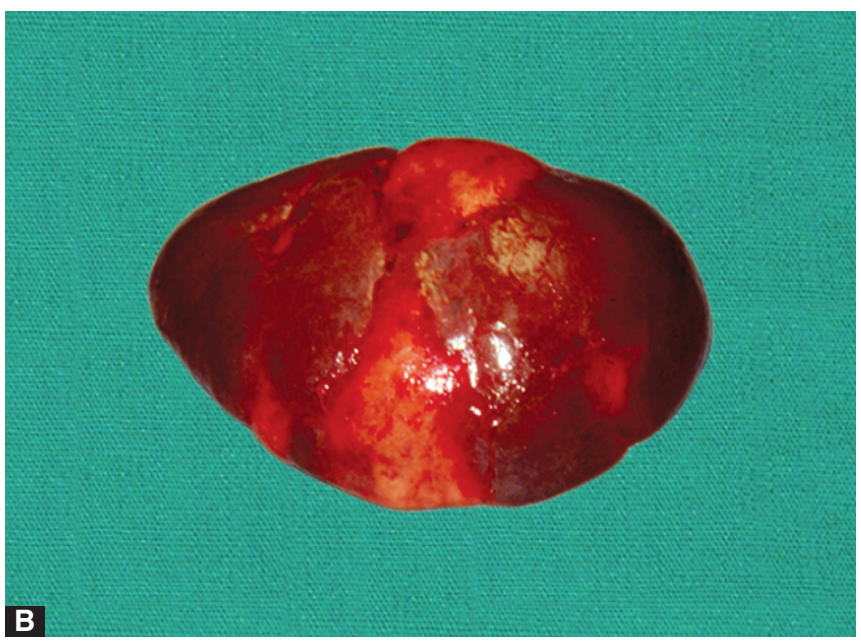

Figs 10A and B: This patient sustained a grade III intrasplenic hematoma following an assault (A). He also used street drugs. Following successful nonoperative therapy, he returned with multiple splenic abscesses, likely due to splenic seeding from his intravenous street drug use (B) 
Successful NOT correlates best with the lower grade of splenic injury and the absence of associated injuries which require laparotomy. ${ }^{25}$ The most important determinant in the decision to initiate and then continue NOT is the clinical picture; age and grade of injury by themselves are less important. A decision to abort NOT is best made on the basis of the need to give a blood transfusion for isolated splenic injury. Now that stable patients are observed without transfusion until the hemoglobin falls to $8 \mathrm{gm} / \mathrm{dl}$, the need to give a blood transfusion indicates that the NOT has failed. ${ }^{2}$ Likewise, when a patient with multiple injuries has to be given more blood than that which would be predicted by the other injuries, laparotomy for splenorrhaphy or splenectomy is indicated.

One of the complications of NOT for patients with a major intrasplenic hematoma is subsequent bacteria seeding of the hematoma and the development of a splenic abscess. ${ }^{2}$

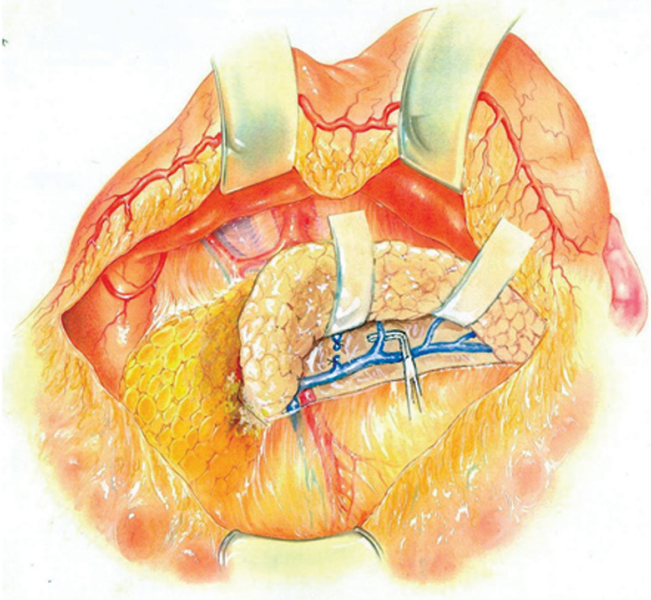

Fig. 11: After freeing the inferior and superior margins of the pancreas, the organ is rotated anteriorly and superiorly to expose the very short and friable branches of the splenic vein entering in to the posterior surface of the pancreas

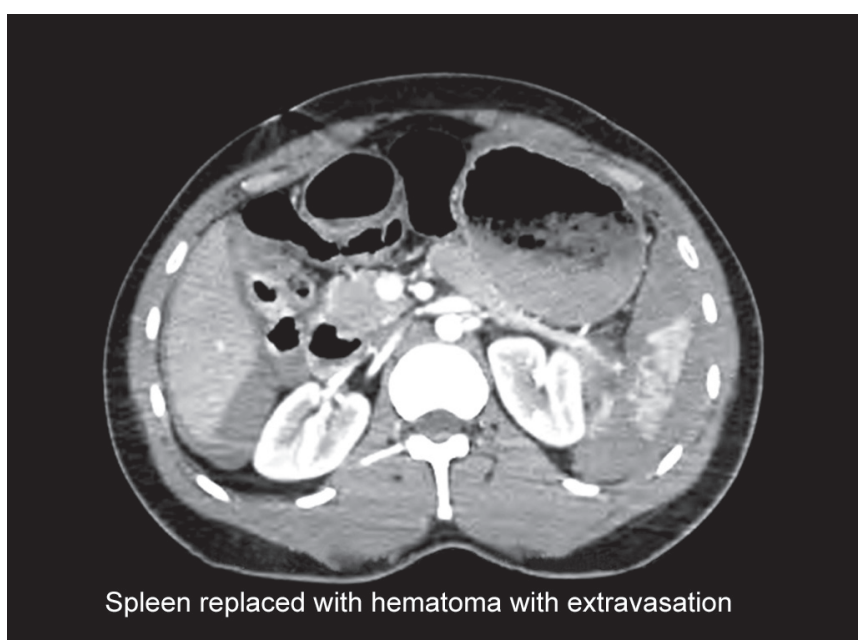

Fig. 12: This patient had a grade IV splenic injury with intrasplenic hematoma, blush, and extravasation. His major associated injury was a fractured patella
This is particularly true in patients who are using street drugs that are often contaminated, thus providing an extra burden of bacteria which becomes sequestered in the spleen (Figs 10A and B). Once this occurs, the optimal treatment is total splenectomy.

\section{OPERATIVE SPLENIC SALVAGE AFTER FAILED NOT}

When a patient fails nonoperative therapy necessitating laparotomy, the same techniques for splenic salvage as described above should be implemented. In retrospect, it now appears that patients who previously had operative splenic salvage are now being successfully treated by the nonoperative approach. ${ }^{25,26}$ Consequently, the high success rate for operative splenorrhaphy when laparotomy is used as the primary treatment falls to about $10 \%$ in patients who fail NOT and require exploration.

\section{OPERATIVE SPLENIC SALVAGE WITH DISTAL PANCREATECTOMY}

The popularity of splenic salvage has extended to patients with pancreatic injury causing ductal disruption in the pancreatic tail. Distal pancreatectomy without splenectomy in this setting is performed by dividing the short branches of the splenic artery and splenic vein as they enter into the pancreas while protecting the terminal branches leading to the hilum. ${ }^{27}$ Initially described for benign or cystic tumors of the body of the pancreas, Pachter reported its successful use for pancreatic trauma. ${ }^{27,28}$ This procedure requires careful mobilization of the lesser omental attachments to the superior border of the pancreas and the transverse colon mesentery attachments to the inferior border of the pancreas while completely mobilizing the splenic flexure of the colon.

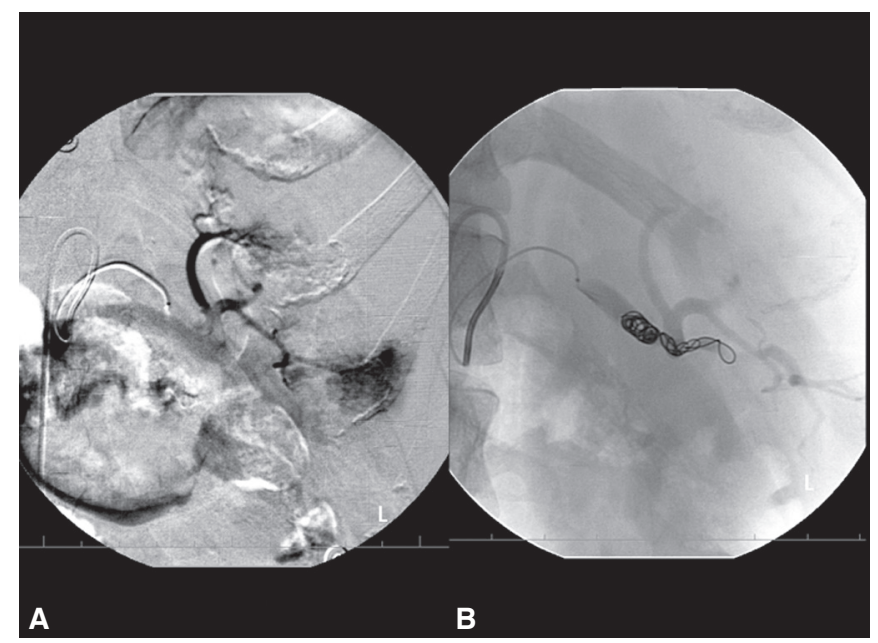

Figs 13A and B: The patient in Figure 12 underwent SAE for the extravasation seen prior to embolization $(A)$ which brought about complete cessation of bleeding after the coils were embolized in the main splenic artery $(B)$ 


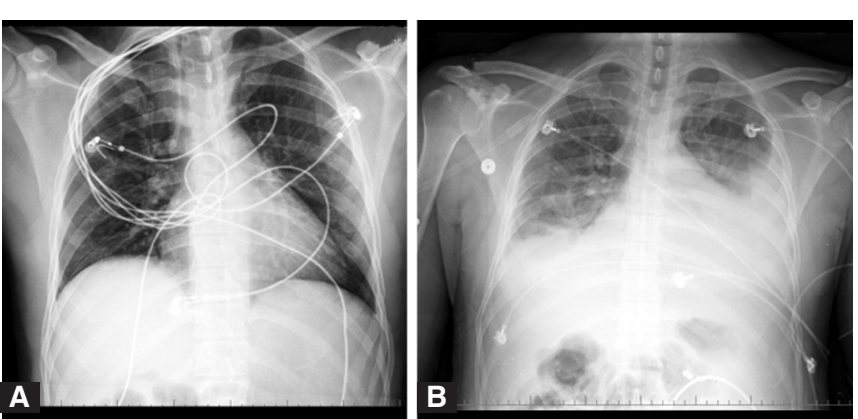

Figs 14A and B: The patient in Figure 12 had a normal chest $X$-ray prior to embolization $(A)$ but following embolization induced infarction, developed a left upper quadrant abscess and left thoracic empyema (B)

The splenic vein and branches are exposed by rotating the inferior of the border of the pancreas anteriorly and superiorly, thus exposing the posterior surface where these short vessels enter the pancreas (Fig. 11). The sequential division of these small veins is best accomplished by moving from the superior mesenteric vein toward the hilum. The accompanying small arterial vessels are much less friable and much easier to control. The pancreas can then be divided by electrocoagulation reinforced by suture ligature or by the GIA stapler. When the main splenic artery has to be divided, continued perfusion of the spleen occurs retrogradely through the short gastric arteries and the posterior gastric artery. This technique is tedious and not without risk; infarction of the lower pole of the spleen is relatively common. Infarction, in turn, may lead to an abscess; early splenectomy prevents this sequence. The author considers distal pancreatectomy without splenectomy for trauma to be meddlesome.

\section{SPLENIC INJURY AND PENETRATING WOUNDS}

The approach to splenic injury following penetrating abdominal injury is somewhat different in that the majority of patients presenting with penetrating wounds have laparotomy for repair of other injuries. ${ }^{3}$ When laparotomy is performed for repair of other injuries, the principles of splenic management are the same. ${ }^{13,29}$ The small grade 1 or grade 2 stab wound or bullet wound can be treated without any definitive surgical procedure if the wound is not bleeding and the patient is stable. Graze injuries to the spleen, or superficial stab wounds which are oozing, can usually be made hemostatic with careful electrocoagulation. Sometimes just placing a pack over the wound for 5 or 10 minutes will stop the bleeding. The major stab wounds and gunshot wounds of the spleen that are amenable to suture splenorrhaphy can be so treated. A perfectly dry splenorrhaphy wound to the spleen does not need drainage, but the author prefers to place a closed drain in proximity to the spleen after performing splenorrhaphy for grade 3 gunshot wounds. ${ }^{2,13}$ The author prefers splenectomy for grade 4 or grade 5 stab wounds or gunshot wounds.

\section{TREATMENT OF THE SPLENIC 'BLUSH'}

The popularity for NOT has led to increased use of interventional techniques to control splenic hemorrhage by splenic artery embolization (SAE). Thus, a stable patient who is being followed nonoperatively but has a splenic blush or dynamic intrasplenic hematoma on contrast imaging is recommended for SAE. ${ }^{30}$ This embolization may be through the main splenic artery or through a branch closer to the extravasation. Dominaga et al showed that immunologic function of the spleen was maintained after SAE. Likewise, several studies have demonstrated that SAE stops bleeding, thereby precluding an emergency operation. ${ }^{31}$

Unfortunately, splenic embolization is not without risk. Even when the SAE is performed through one of the segmental branches, splenic infarction may result. This leads to sudden severe pain, which is associated with leukocytosis and fever. The symptoms of this SAE infarction are totally reversed by prompt splenectomy. Shih et al have suggested that the embolization alters the immune response of mononuclear cells, which thereby increases the susceptibility for a splenic infection. ${ }^{32}$ Blind adherence to the need for NOT of blunt splenic rupture may lead to a sequence of infectious events that ultimately are fatal. An example of this is provided.

This young man presented as a restrained passenger after a high-speed MVC. He had stable vital signs but was amnestic to the event. Injuries included a patellar fracture and type 4 splenic rupture with intrasplenic hematoma, blush, and extravasation (Fig. 12). The extravasation was treated with SAE with coils placed in the distal splenic artery (Figs 13A and B). He received 2 units RBCs for anemia on postinjury day 3 when he had operative fixation of his patella. By postoperative day 4, he had a left pleural effusion with fever and tachycardia by day 6. A thoracentesis yielded $300 \mathrm{ml}$ of fluid, which was negative on culture, although he continued to complain of left upper quadrant pain; his white count rose to 21,000 . By day 8 , he had atelectasis, tachycardia, and leukocytosis. Re-imaging studies demonstrated a $3 \mathrm{~mm}$ pseudoaneurysm of an inferior branch of the splenic artery; this was coiled, and he was discharged home on postinjury day 13. During his hospitalization, he received 4 units RBCs, had three CTs and two angiograms with embolization. Two weeks later, he had leukocytosis $(22,600)$ and thrombocytosis $(905,000)$. By six weeks, he was admitted to the medical service for atelectasis and received broad spectrum antibiotics. Aspiration of the left upper quadrant yielded $60 \mathrm{ml}$ of thick pus. The serum creatinine rose from 1.5 to $4.7 \mathrm{mg} / \mathrm{dl}$; tachycardia continued. Over the next week, his creatinine rose to $10.9 \mathrm{mg} / \mathrm{dl}$ and the nephrotoxic antibiotics were changed. 
Thoracentesis confirmed empyema, which contributed to a cardiac arrest, from which he was resuscitated (Figs 14A and B). Laparotomy on day 52 revealed $1.5 \mathrm{~L}$ of ascites and a fragmented necrotic abscessed spleen, which was removed in pieces. Three days later, he had a thoracotomy with decortication, tracheostomy for long-term ventilatory support, and finally, on hospital day 100 , he was declared comfort care and died shortly thereafter. All of this could have been prevented by splenectomy on day 3 .

\section{OPERATIVE SPLENORRHAPHY/SPLENECTOMY}

The successes achieved with SAE have caused the pendulum to swing too far from operative therapy. ${ }^{26}$ The attractiveness of NOT has caused an excessive use of SAE. The above patient would have done much better had the surgeon realized that the administration of 2 units of blood at the time of the operative fixation of the patella fracture indicates that the bleeding is too excessive for NOT. Further surgical intervention was indicated when the signs and symptoms of splenic infarction sequelled SAE. The additional detriment to the routine use of SAE has been the loss of technical skill by young trauma surgeons, who rarely do splenectomy for injury. This creates a technical gap for patients in need of early splenectomy. Recently, one of our midlevel trauma surgeons took four hours to help a senior resident remove a ruptured spleen; this should occur in one hour. This lack of experience in splenectomy will soon be identified as a major problem in the care of patients with ruptured spleen.

\section{COMPLICATIONS OF SPLENECTOMY}

The most frequent and feared complication of splenectomy is postoperative bleeding. ${ }^{2,29}$ This bleeding usually arises from the short gastric vessels followed by the unnamed vessels coming from the diaphragm and the splenic hilar vessels. Since the greatest attention at hemostasis is usually directed to the splenic artery and vein, this seldom is the source of postoperative bleeding. Once bleeding occurs, the intraperitoneal clot should be evacuated rapidly and a number of packs placed in the left upper quadrant. By slowly removing the packs from the diaphragm toward the stomach and then toward the small vessels coming from the splenic flexure of the colon, one can sequentially identify bleeders and obtain hemostasis.

Visceral fistula following splenectomy can occur from the proximal stomach where the short gastric vessels were taken down, from the tail of the pancreas if the clamp on the splenic vessels incorporates some pancreatic tissue, and from the splenic flexure of the colon if the clamps getting the short vessels going to the lower pole are placed too close to the colon. Care in identifying where the clamps are placed in these three areas will eliminate the problems with a splenic fistula. The contused greater curve should be inverted. Once a fistula does occur, one needs to achieve external drainage and then determine optimal treatment.

Thrombocytosis occurs routinely following splenectomy. When the patients are seen in an office setting, the platelet count will be over one million. These platelets are old and dysfunctional, so that anticoagulation is not indicated.

A splenic abscess which occurs following splenectomy is usually the result of inadequate hemostasis at the time of surgery or spillage from other organ injuries. When multiple other organ injuries are present, particularly colon injuries, the author prefers to leave a left upper quadrant drain. In the absence of hollow-visceral injuries, a left upper quadrant abscess is quite rare, so that drains to identify an early abscess or a fistula are not indicated.

\section{THE SPLENIC BLUSH}

The advantages in radiologic intervention with various types of SAE has prompted intervention in stable patients who have a blush after blunt trauma. Certainly, SAE can be helpful for patients scheduled for splenorrhaphy or splenectomy. However, when the patient with a splenic blush is asymptomatic, SAE is potentially dangerous. This was brought to the author's attention when a middle-aged woman had a blunt rupture of the abdomen and chest following an MVC. She had two left-sided rib fractures and a type 3 splenic injury. She was observed nonoperatively at her primary hospital and then referred 1 week after injury for treatment of the splenic blush. She was scheduled electively to have SAE as an outpatient. Thirty-six hours after SAE, she returned to the hospital with left upper quadrant pain, fever, malaise, and leukocytosis. She then underwent open splenectomy, felt great the next day, and was discharged home two days later. The splenic specimen showed infarction. The author recommends that the asymptomatic patient with a splenic blush be observed nonoperatively without SAE. ${ }^{1,2}$

\section{ST CENTURY GUIDELINES FOR CARE OF SPLENIC INJURY}

Most patients with blunt rupture to the spleen can be treated nonoperatively. Even patients with major injuries who are stable and do not require blood transfusions are candidates for NOT. The decision to operate for any severity of splenic injury should be based upon the patient's clinical examination, vital signs, and lack of need for blood transfusion thought to be related to the splenic hemorrhage. The treatment of penetrating wounds follows the same guidelines, although laparotomy for other reasons is much 
more frequently performed. A penetrating wound in the left lower thorax associated with a hemothorax is often associated with a perforated diaphragm and an unrecognized splenic injury. This can be diagnosed by laparoscopy in order to repair the diaphragmatic injury and prevent subsequent herniation. The splenic injury can be treated according to the above guidelines.

When a patient has splenic injury in association with other injuries, one must make a best estimate as to when the need for blood transfusions exceeds that which can be predicted by the other injuries. When that occurs, intervention is indicated. SAE can be attempted in the stable patient, whereas the author recommends operative intervention and definitive splenorrhaphy or splenectomy in unstable patients. When splenic artery embolization is used, any evidence of splenic infarction should be promptly treated with open splenectomy.

The different techniques for splenorrhaphy are well established. The author believes that any spleen which cannot be appropriately dealt with by splenorrhaphy or partial splenectomy should be removed. Placing a mesh around a badly injured spleen is probably meddlesome. The incidence of OPSI is very low, particularly when one is given a polyvalent vaccine and appropriately covered with antibiotics.

The use of splenic replantation following total splenectomy is no longer recommended; although the replanted splenic tissues survive, the histology show that the parafollicular lymphoid cells do not regenerate and these cells are the source of the immune responses of the spleen. Likewise, the diced spleen, which is replanted, infarcts and may necessitate emergency removal.

Following splenorrhaphy or NOT for a ruptured spleen, splenic wound healing is quite good and is better than that seen with abdominal fascia. Therefore, concerns about preventing a person who has had an injured spleen from returning to activity should be based upon the guidelines that one would use for the midline fascia rather than the injured spleen itself.

The recognition that OPSI occurs necessitates the need for protection following partial or total splenectomy. ${ }^{8}$ The polyvalent vaccine should be given prior to discharge, and then a booster shot should be given while the patient is being followed in the outpatient department. Likewise, the patient should be aware of his/her splenic state and arrange to get antibiotic therapy if some infection occurs.

Finally, the trauma surgeon needs to maintain his skills in performing splenectomy. The overuse of SAE will lead to an inappropriately high incidence of splenic infarction. Likewise, it deprives the surgeon of the technical skills of performing a rapid and safe splenectomy.

\section{REFERENCES}

1. Lucas CE, Ledgerwood AM. Splenic preservation. In Mastery of Surgery. 5th ed. In: JE Fischer, KI Bland, editors. Lippincott Williams and Wilkins. 2007;p.1670-1678.

2. Lucas CE. Splenic trauma: choice of management. Ann Surg 1991;213(2):98-112.

3. Saxe JM, Hayward SR, Lucas CE, Muz J, Ledgerwood AM, Lucas D, Joseph A, Lucas W. Splenic reimplantation does not affect outcome in chronic canine model. Am Surg 1994;60: 674-680.

4. Morris DH, Bullock FD. The importance of the spleen in resistance to infections. Ann Surg 1919;70:513-521.

5. Bailey H. Traumatic rupture of the normal spleen. Br J Surg 1927; 15:50-56

6. Pfeiffer DB, Smith CM. Late result of splenectomy for traumatic rupture of the spleen. Ann Surg 1924;80:562-568.

7. McIndoe AH. Delayed hemorrhage following traumatic rupture of the spleen. Br J Surg 1932;20:249-268.

8. King H, Schumacker HB Jr: Splenic studies: 1. susceptibility to infection after splenectomy performed in infancy. Ann Surg 1952;136:239-242.

9. Hoefnagel R. Susceptibility of infection after splenectomy performed in childhood. Clin Proc Child Hosp 1956;12:48-55.

10. Smith CH, Erlandson M, Schulman I, Stern G. Hazard of severe infections in splenectomized infants and children. Am J Med 1957;22:390-404.

11. Moore EE, Cogbill TH, Jurkovich GJ, et al. Organ injury scaling: spleen and liver (1994 revision). J Trauma 1995;38:323.

12. Morgenstern L, Shapiro ST. Techniques for splenic preservation. Arch Surg 1979;114:449-454.

13. Morgenstern L, Skandalakis JE. Anatomy and embryology of the spleen. In: Hiatt JR, Phillips EH, Morgenstern L, editors. Surgical Diseases of the Spleen. New York, Springer. 1997.

14. Okinaga G, Giebink GS, Rich RH. The effect of partial splenectomy on experimental pneumococcal bacteremia in an animal model. J Pediat Surg 1981;16:717-724.

15. Resende V, Petroianu A. Functions of the splenic remnant after subtotal splenectomy for treatment of severe splenic injuries. Am J Surg 2003;185(4):311-315.

16. Feliciano DV, Bitondo CG, Mattox KL. A four-year experience with splenectomy versus splenorrhaphy. Ann Surg 1985;201: 568-575.

17. Shennib H, Chiu RC, Mulder DS. The effects of splenectomy and splenic implantation on immune function. J Trauma 1983;23: 7-12.

18. Nicholson S, Hutchinson GH, Hawkins T, et al. Successful splenosis following autologous splenic implantation. JR Coll Surg Edinb 1986;31:67-71.

19. Witte CL, Witte MH, McNeil GC. Splenic salvage quantified by uptake of heat-damaged radiolabeled red blood cells. Am J Surg 1988;155:303-310.

20. Moore GE, Stevens RE, Moore EE, et al. Failure of splenic implants to protect against fatal postsplenectomy infection. Am J Surg 1983;146:413.

21. Dulchavsky SA, Lucas CE, Ledgerwood AM, et al. Wound healing of the injured spleen with and without splenorrhaphy. J Trauma 1987;27:1155-1160.

22. Tom WW, Howells GA, Bree RL, et al. A non-operative approach to the adult ruptured spleen sustained from blunt trauma. Arch Surg 1985;51:367-371. 
23. Villalba MR, Howells GA, Lucas RJ, et al. Nonoperative management of the adult ruptured spleen. Arch Surg 1990;125(7):836-839.

24. Meguid AA, Bair HA, Howles GA, et al. Prospective evaluation of criteria for the nonoperative management of blunt splenic trauma. Am Surg 2003;69:238-243.

25. Feliciano D. American association for the surgery of trauma spleen study (AAST) group. Nonoperative management of the injured spleen: a prospective study from the American Association for the Surgery of Trauma Multi-Institutional Trial Committee. Paper presented at 63rd Annual Meeting of the American Association for the Surgery of Trauma; September 2004; Hawaii.

26. Smith J, Ahmen, $\mathrm{S}$, Cook $\mathrm{CH}$, et al. Blunt splenic injuries: have we watched long enough? J Trauma 2008;64(3):656-663.

27. Pachter HL, Hofstetter SR, Liang HG, et al. Traumatic injuries to the pancreas: the role of distal pancreatectomy with splenic preservation. J Trauma 1989;29(10):1352-1355.

28. Warshaw AL. Conservation of the spleen with distal pancreatectomy. Arch Surg 1988;123:550-553.
29. Sherman R. Prospective in management of trauma to the spleen: 1979 presidential address. American Association for the Surgery of Trauma. J Trauma 1980;20:1-13.

30. Hann JM, Marmery H, Shanomerganathan K, et al. Experience with splenic main coil embolization and significance of new or persistent pseudoaneurysm: reembolize, operate, or observe. J Trauma 2007;63:615-619.

31. Haan JM, Biffl W, Knudson M, et al. Splenic embolization revisited: a multicenter review. J Trauma 2004;56(3):542-547.

32. Shih HC, Wang CY, Wen YS, Wu JK, Huang MS, Huang $\mathrm{CL}$, Leech. Splenic artery embolization aggravate endotoxin hyporesponse of peripheral blood mononuclear cells in patients with splenic injury. J Trauma 2010;68:532-537.

\section{ABOUT THE AUTHOR}

\section{Charles E Lucas}

Professor, Department of Surgery, Detroit Receiving Hospital, Wayne State University, Detroit, Michigan, USA, e-mail: clucas@med. wayne.edu 